\title{
FACTORES SOCIOCULTURALES, PERSONALES E INSTITUCIONALES RELACIONADOS A LA ACEPTABILIDAD DE SOLUCIONES REHIDRATANTES ORALES, EN USUARIOS DE ESTABLECIMIENTOS DE SALUD DEL DISTRITO DE COMAS. LIMA-PERÚ 2016
}

\author{
Walter Gómez Gona les¹, Asunciona Huaman Cruz², Johana Ramirez Cerna³ ${ }^{3}$ María Auqui Canchari
}

\begin{abstract}
RESUMEN
Objetivo. Establecer los factores socioculturales, personales e institucionales relacionados a la aceptabilidad de soluciones rehidratantes orales, en usuarios de establecimientos de salud del Distrito de Comas. Lima-Perú 2016. Materiales y métodos. Estudio de diseño descriptivo correlacional. La población estuvo constituida por pacientes con deshidratación leve-moderada atendidos en los establecimientos de salud del Distrito de Comas, conformada por 06 Centros de Salud y 01 Puesto de Salud, se incluyeron 101 pacientes y se realizó el llenado de un cuestionario ad hoc para el estudio. El procesamiento, análisis de datos y contrastación de la hipótesis se realizó con el programa estadístico SPSS versión 23. Resultados. De 101 casos de pacientes con diarrea con deshidratación, 66 (65\%) fueron de sexo femenino y 35 (35\%) de sexo masculino, edad promedio 10,99 años, tiempo de enfermedad promedio de 2,14 días y el grado de instrucción predominante fue secundaria completa $38(38 \%)$, superior $20(20 \%)$ y secundaria incompleta $15(15 \%)$. Los factores que presentan correlación estadísticamente significativa con la aceptabilidad de soluciones rehidratantes orales, fueron el factor sociocultural $r=0,781$ y valor $p 0,000$, con las variables grado de instrucción secundaria completa y superior, procedencia sierra, ingiere como primera opción soluciones hidratantes preparadas diferentes a las S.R.O. con electrolitos listas para tomar; el factor personal $r=0,659$ - valor $p 0,026$, con las variables edad de 0 a 5 años, no ingiere toda la S.R.O. para preparar prescrita, acepta la solución preparada de S.R.O. y preferencia por otros hidratantes orales preparados; el factor institucional no obtuvo relación estadísticamente significativa $r=$ 0,223 - valor $p$ 0,509. Conclusiones. Los factores relacionados con la aceptabilidad de soluciones rehidratantes orales, fueron el grado de instrucción secundaria completa y superior, procedencia sierra, ingiere como primera opción soluciones hidratantes preparadas diferentes a las S.R.O. con electrolitos listas para tomar, edad de 0 a 5 años, no ingiere toda la solución de S.R.O. prescrita, no acepta la solución de S.R.O. para preparar y preferencia por otros hidratantes orales preparados.
\end{abstract}

Palabras Clave: Sales de rehidratación oral; Factores; Aceptabilidad (Fuente: Decs BIREME).

\section{SOCIOCULTURAL, PERSONAL AND INSTITUTIONAL FACTORS RELATED TO THE ACCEPTABILITY OF ORAL REHYDRATION SOLUTIONS IN USERS OF HEALTH FACILITIES IN THE DISTRICT OF COMAS. LIMA-PERU 2016}

\begin{abstract}
Objective. To establish the sociocultural, personal and institutional factors related to the acceptability of oral rehydration solutions in users of health facilities in the Comas District. Lima-Perú 2016. Materials and methods. A descriptive correlational design study. The population consisted of patients with mild-moderate dehydration treated at Comas District health facilities, comprising 06 Health Centers and 01 Health Care Unit, 101 patients were included and an ad hoc questionnaire was completed for the studio. Results. From 101 cases of patients with diarrhea with dehydration, $66(65 \%)$ were female and $35(35 \%)$ were female. (38\%), upper $20(20 \%)$ and incomplete secondary school $15(15 \%)$ were secondary school students. The factors that present a statistically significant correlation with the acceptability of oral rehydration solutions were the sociocultural factor $r=$ 0.781 and $p$ value 0.000 , with the variables degree of secondary education complete and superior, sawing source, ingested as a first option prepared moisturizing solutions different from the ORS with electrolytes ready to drink; the personal factor $r=$ 0.659 - $p$ value 0.026 , with the variables age from 0 to 5 years, does not ingest the entire S.R.O. to prepare prescribed, accept the prepared solution of S.R.O. and preference for other oral oral hydrates; the institutional factor did not obtain statistically significant relation $r=0.223$ - value $p$ 0.509. Conclusions. The factors related to the acceptability of oral rehydration solutions, were the degree of secondary education complete and superior, sawing source, ingested as a first option prepared moisturizing solutions different to the S.R.O. with electrolytes ready to drink, age 0 to 5 years, does not ingest the entire solution of S.R.O. does not accept the solution of S.R.O. to prepare and preference for other prepared oral moisturizers.
\end{abstract}

Key Words: Oral rehydration salts; Factors; Acceptability (Source: MeSH NLM).

\footnotetext{
Lic.en Enfermería. Doctor en Ciencias de la Salud, Facultad de Medicina, Universidad Nacional Mayor de San Marcos. Profesor Universidad Alas Peruanas, Escuela de Post Grado. Lima-Perú. Profesor Universidad Andina del Cusco, Escuela de Post Grado. Cusco-Perú.

Lic. en Enfermería. Coordinadora de Cuidado Integral-Red de Salud Túpac Amaru, Dirección Regional de Salud Lima Norte-MINSA. Lima-Perú. Doctor en Educación. Directora de Maestrias y Segundas Especialidades, Universidad Alas Peruanas. Lima-Perú.

Lic. en Obstetricia. Grado académico de Doctor. Coordinadora de Segunda Especialidad, Universidad Alas Peruanas, Escuela de Post Grado. Lima-Perú.

Citar como: Gómez W, Huaman A, Ramirez J, Auqui M. Factores socioculturales, personales e institucionales relacionados a la aceptabilidad de soluciones rehidratantes orales, en usuarios de establecimientos de salud del distrito de Comas. Lima-Perú 2016. Rev Peru Investig Matern Perinat $2018 ; 7(1): 31-6$. DOI https://doi.org/10.33421/inmp.2018107
}

Recibido: 10-06-18 Aprobado: 30-06-18 


\section{INTRODUCCIÓN}

La enfermedad diarreica aguda (EDA), continúa siendo un problema importante de salud pública al ser una de las principales causas de muerte en menores de 5 años, por el elevado número de casos que se presentan anualmente y por los gastos que genera el tratamiento médico general o específico. Se estima que cada año la diarrea mata a 760.000 niños menores de 5 años. Ocho de cada 10 de estas muertes ocurren en los primeros dos años de vida ${ }^{1,2}$. Pese a que la gran maø ría de los a adros diarreio $s$ resuelve en corto tiempo con medidas básicas (empleo de SRO), un porcentaje de los niños sufre complicaciones graves, como la deshidratación que puede llevar a la muerte. A través del reconocimiento oportuno de datos de deshidratación, el manejo adecuado, así como la identificación de factores de riesgo que podrían empeorar el curso de la enfermedad, el personal de salud puede también contribuir a evitar sus complicaciones.

La estrategia principal para reducir la mortalidad infantil por diarrea ha sido y sigue siendo la Terapia de Rehidratación Oral (TRO). La tasa de uso de la TRO, se refiere generalmente al uso de la SRO o los líquidos caseros recomendados (SRO/LCR), y es definida como la proporción de niños menores de cinco años con diarrea que reciben un suplemento de líquidos y alimentación o ntinuada ${ }^{3}$. Si la tasa de uso de la TRO es difícil de valorar, la tasa de uso eficaz, es aún más problemática, es reconocido que el uso ineficaz de la TRO tanto sobres o mo mez as a seras o líquidos a seros es un gran obstáculo. Norbert Hirschhorn, uno de los pioneros de la TRO, admite que está bastante claro que muchos niños no rec ben lo que nee sitan int uso a ando los sobres y líquidos caseros son conocidos y están disponibles ${ }^{4}$. Las estimaciones sobre el uso eficaz varían mucho, pero, por término medio, se piensa que, en la actualidad, sólo una tercera parte de las veces se usa correctamente.

La TRO con productos listos para tomar, está indicada para prevenir la deshidratación, rehidratar y mantener el estado de hidratación, independientemente de la edad del paciente, del agente etiológico y de los valores iniciales de sodio sérico tan igual que los sobres rehidratantes, con la diferencia de no realizar la preparación ya que los rehidratantes orales están listos para tomar, siendo las S.R.O. no muy bien tolerados por los pacientes pediátricos por el sabor salado. Por tanto, el esfuerzo de búsqueda del rehidratante oral listo para tomar que cubra las necesidades del paciente, que nos ayude a restablecer rápidamente el equilibrio hidroelectrolítico en nuestro organismo para evitar complicaciones y probablemente la muerte por deshidratación grave, es el propósito básico del presente trabajo de investigación, focalizado en establecimientos de salud del distrito de Comas, generando la evidencia científica que nos permita establecer los factores que puedan estar relacionados a la aceptabilidad de soluciones rehidratantes orales, en estableć mientos de salud del Distrito de Comas.
El objetivo de la investigación es establecer los factores socioculturales, personales e institucionales relacionados a la aceptabilidad de soluciones rehidratantes orales, en usuarios de establec mientos de salud del Distrito de Comas. Lima-Perú 2016.

\section{MATERIALES Y MÉTODOS}

Tipo de Estudio. El estudio realizado fue una investigación no experimental, transversal porque se hizo una sola medición de las variables en estudio y en un solo momento, se enmarcó dentro del diseño correlacional (5), porque se describió las relaciones entre los factores socioculturales, personales e institucionales relacionados a la aceptabilidad de soluciones rehidratantes orales.

En consecuencia, por la naturaleza de la investigación, se adopta este diseño, que se diagrama de la siguiente forma:

\section{Esquema}
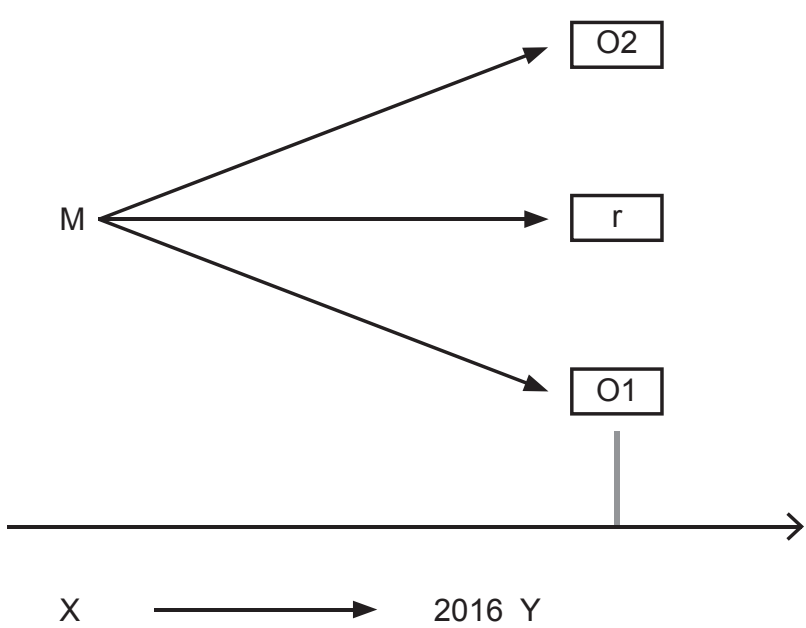

- M: Usuarios establecimientos de salud del Distrito de Comas

- 01: Factores socioculturales, personales e institucionales

- r: Probable correlación

- O2: Aceptabilidad de soluciones rehidratantes orales

Población y muestra. La población estuvo constituida por el $100 \%$ de casos atendidos por enfermedad diarreica aguda, en un día de semana del mes de Noviembre del 2016 seleccionado de forma aleatoria; los establecimientos de salud incluidos en el estudio fueron Centro de Salud Laura Rodríguez, Centro de Salud Progreso, Centro de Salud Año Nuevo, Centro de Salud Carmen Medio, Centro de Salud Milagros Fraternidad, Centro de Salud Primavera y Puesto de Salud Geranios, siendo como sigue el total de casos incluidos por establecimiento: 


\begin{tabular}{lc}
\hline \multicolumn{1}{c}{$\begin{array}{c}\text { Establecimientos de Salud } \\
\text { Distrito de Comas }\end{array}$} & Muestra \\
\hline C.S. Laura Rodríguez & 31 \\
P.S. Geranios & 20 \\
C.S. Progreso & 10 \\
C.S. Año Nuevo & 10 \\
C.S. Carmen Medio & 10 \\
C.S. Milagros Fraternidad & 10 \\
C.S. Primavera & 10 \\
Total & $\mathbf{1 0 1}$ \\
\hline
\end{tabular}

No se consideró el cálculo de tamaño muestral, ya que se trabajó con todos los casos que cumplieron los criterios de inclusión y exclusión, el muestreo fue por conveniencia.

- Criterios de Inclusión: Pacientes de 01 año a más, de ambos sexos, con el diagnóstico de enfermedad diarreica aguda con deshidratación, atendido en alguno de los estableć mientos de salud int uidos en el estudio y que acepten participar en el estudio.

- Criterios de Exclusión: Pacientes menores de 01 año a más, de ambos sexos, con el diagnóstico de enfermedad diarreica aguda con deshidratación y shock y/o sin deshidratación, atendido en alguno de los establec mientos de salud int uidos en el estudio y que no acepten participar en el estudio.

Técnicas e instrumentos de recolección de datos. La técnica empleada fue la encuesta y el instrumento utilizado fue la guía de encuesta, que fue diseñado para esta investigac ón de au erdo a las variables que se tomaron en cuenta, la validez del instrumento de recolección de datos se estableció por juicio de expertos, constituido por 05 expertos profesionales de la salud especialistas en la línea de investigać ón. Procesamiento y análisis estadístico de los datos. Para el procesamiento de los datos se hizo uso del programa estadístico SPSS versión 25 para Windows, se hizo uso de la estadística descriptiva. Aspectos Éticos. Se ha tenido en a enta o nsentimiento informado verbal del usuario si es mayor de 18 años y si es menor el consentimiento informado verbal de los padres del menor de edad incluido en el estudio. Se ha tenido en cuenta para el consentimiento informado verbal los 4 principios de la Bioética: No Maleficencia, justicia, beneficencia y autonomía.

\section{RESULTADOS}

La tabla 1, muestra del total de usuarios encuestados el $65 \%(66 / 101)$ fue de sexo femenino y el $35 \%$ (35/101) fue de sexo masculino; respecto a la edad de los usuarios un $49 \%$ (50/101) fluctúan entre 1 a 4 años, un 32\% (32/101) fueron mayores de 20 años, un $16 \%$ (16/101) fueron de 5 a 10 años y un 3\% (3/101) fueron de 11 a 20 años; en cuanto al grado de instrucción predomina la categoría secundaria completa con un $37 \%$ (38/101), seguido por educación superior con un $20 \%(20 / 101)$, secundaria incompleta con un $15 \%$ (15/101), primaria completa con un $11 \%(11 / 101)$ y analfabeto con un $3 \%(3 / 101)$.

La tabla 2, muestra la significancia estadística bivariada de los factores socioculturales, personales e institucionales relacionados a la aceptabilidad de soluciones rehidratantes orales, en usuarios de establecimientos de salud del Distrito de Comas. LimaPerú 2016; siendo el factor sociocultural $r=0,781$ y valor p 0,000 , con las variables grado de instrucción secundaria completa y superior, procedencia sierra, ingiere como primera opción soluciones hidratantes preparadas diferentes a las S.R.O. con electrolitos listas para tomar; el factor personal $r=0,659$ - valor $p 0,026$, con las variables edad de 0 a 5 años, no ingiere toda

Tabla 1. Características sociodemográficas de los usuarios de los establecimientos de salud del distrito de Comas, 2016

\begin{tabular}{|c|c|c|}
\hline Edad & $\mathbf{N}^{\circ}$ & $\%$ \\
\hline Femenino & 66 & 65 \\
\hline Masa lino & 35 & 35 \\
\hline \multicolumn{3}{|l|}{ Sexo } \\
\hline 1 a 4 años & 50 & 49 \\
\hline 5 a 10 años & 16 & 16 \\
\hline 11 - 20 años & 3 & 3 \\
\hline$>$ de 20 años & 32 & 32 \\
\hline \multicolumn{3}{|l|}{ Nivel de instrucción de la madre } \\
\hline Analfabeto & 5 & 5 \\
\hline Primaria incompleta & 11 & 11 \\
\hline Primaria completa & 11 & 11 \\
\hline Secundaria incompleta & 15 & 15 \\
\hline Secundaria completa & 38 & 37 \\
\hline Superior & 20 & 20 \\
\hline
\end{tabular}

Fuente: Proyecto FRAS.R.O. 
Tabla 2. Análisis Bivariado - Factores socioculturales, personales e institucionales relacionados a la aceptabilidad de soluciones rehidratantes orales, en usuarios de establecimientos de salud del Distrito de Comas. Lima-Perú 2016.

\begin{tabular}{|c|c|c|c|c|}
\hline Factores & Categorías & Valor $p$ & r spearman & IC (95\%) \\
\hline \multicolumn{5}{|l|}{ Socioculturales } \\
\hline \multirow{6}{*}{ Grado de instruc ón } & Analfabeto & 0.524 & 0.292 & $0.365-1.575$ \\
\hline & Primaria incompleta & 0.457 & 0.485 & $0.437-1.525$ \\
\hline & Primaria completa & 0.205 & 0.416 & $0.801-2.790$ \\
\hline & Secundaria incompleta & 0.598 & 0.468 & $0.267-2.141$ \\
\hline & Secundaria completa & 0,038 & 0,751 & $1.082-4.234$ \\
\hline & Superior & 0,012 & 0,696 & $1.043-7.765$ \\
\hline \multirow{3}{*}{ Proe denic a } & Costa & 0.485 & 0.083 & $0.394-1.556$ \\
\hline & Sierra & 0.019 & 0,816 & $1.132-4.345$ \\
\hline & Selva & 0.416 & 0.163 & $0.529-4.618$ \\
\hline \multirow{2}{*}{$\begin{array}{l}\text { Ingiere como primera opción } \\
\text { soluciones hidratantes preparadas } \\
\text { diferentes a las S.R.O. con } \\
\text { electrolitos listas para tomar }\end{array}$} & Si & 0,000 & 0,703 & $3.775-16.355$ \\
\hline & No & 0,752 & 0,347 & $0.595-2.055$ \\
\hline \multirow{2}{*}{$\begin{array}{l}\text { Usuario con deshidratación es } \\
\text { atendido en a sa o llevado al Centro } \\
\text { de Salud }\end{array}$} & Casa & 0,065 & 0,411 & $0.942-5.046$ \\
\hline & Centro de Salud & 0,468 & 0,055 & $0.395-7.414$ \\
\hline \multirow{2}{*}{$\begin{array}{l}\text { Donde prepara y/o consume el } \\
\text { rehidratante oral }\end{array}$} & Casa & 0,444 & 0,340 & $0.685-4.946$ \\
\hline & Centro de Salud & 0,366 & 0,056 & $0.553-2.017$ \\
\hline \multirow{4}{*}{$\begin{array}{l}\text { Hábitos de consumo de líquidos del } \\
\text { usuario }\end{array}$} & Gaseosas & 0.339 & 0,468 & $0.612-4.123$ \\
\hline & Bebidas energizantes & 0.874 & 0,398 & $0.583-2.138$ \\
\hline & Bebidas deportivas & 0.740 & 0,133 & $0.512-1.769$ \\
\hline & Agua & 0.201 & 0,411 & $0.353-1.246$ \\
\hline \multirow{2}{*}{ Sabe preparar las S.R.O. } & Positivo & 0.292 & 0,322 & $0.608-5.104$ \\
\hline & Negativo & 0.485 & 0,156 & $0.394-1.556$ \\
\hline \multirow{2}{*}{$\begin{array}{l}\text { Usuario cuenta con los servicios de } \\
\text { agua y desagüe }\end{array}$} & Si & 0.416 & 0,481 & $0.529-4.618$ \\
\hline & No & 0.468 & 0,122 & $0.395-7.414$ \\
\hline \multicolumn{5}{|l|}{ Personales } \\
\hline Edad & $\begin{array}{l}1 \text { a } 4 \text { años } \\
5 \text { a } 10 \text { años } \\
11 \text { a } 20 \text { años } \\
>20 \text { años }\end{array}$ & $\begin{array}{l}0.001 \\
0.339 \\
0.874\end{array}$ & $\begin{array}{l}0,855 \\
0,115 \\
0,429\end{array}$ & $\begin{array}{r}1.132-4.345 \\
0.612-4.123 \\
0.583-2.138\end{array}$ \\
\hline Tiempo de enfermedad & $\begin{array}{l}<\text { día } \\
1 \text { a } 3 \text { días } \\
4 \text { a } 7 \text { días }\end{array}$ & $\begin{array}{l}0.740 \\
0.201 \\
0.130\end{array}$ & $\begin{array}{l}0,372 \\
0,245 \\
0,381\end{array}$ & $\begin{array}{l}0.512-1.769 \\
0.353-1.246 \\
0.292-6.239\end{array}$ \\
\hline \multirow{2}{*}{$\begin{array}{l}\text { Ingiere toda la solución preparada } \\
\text { de S.R.O. prescrita }\end{array}$} & $\mathrm{Si}$ & 0.635 & 0,132 & $0.839-3.813$ \\
\hline & No & 0.017 & 0,699 & $0.179-0.842$ \\
\hline \multirow{2}{*}{$\begin{array}{l}\text { Acepta la solución preparada de } \\
\text { S.R.O. }\end{array}$} & Si & 0,571 & 0,123 & $1.017-4.517$ \\
\hline & No & 0,007 & 0,794 & $0.815-4.773$ \\
\hline \multirow{2}{*}{$\begin{array}{l}\text { Preferencia por otros hidratantes } \\
\text { orales preparados. }\end{array}$} & Si & 0,001 & 0,813 & $0.625-2.161$ \\
\hline & No & 0,225 & 0,333 & $1.323-4.724$ \\
\hline \multicolumn{5}{|l|}{ Institucionales } \\
\hline \multirow{5}{*}{$\begin{array}{l}\text { Personal de salud que atiende en } \\
\text { primera instancia al paciente con } \\
\text { deshidrataic ón }\end{array}$} & Medio & 0,524 & 0,019 & $0.365-1.575$ \\
\hline & Enfermera & 0,457 & 0,224 & $0.437-1.525$ \\
\hline & Obstetriz & 0,205 & 0,421 & $0.801-2.790$ \\
\hline & Técnico de Enfermeria & 0,598 & 0,116 & $0.267-2.141$ \\
\hline & Otro personal & 0,292 & 0,361 & $0.608-5.104$ \\
\hline \multirow{4}{*}{ Área de atenc ón del usuario } & Consultorio ek erno & 0,485 & 0,228 & $0.394-1.556$ \\
\hline & Emergenć a & 0,416 & 0,139 & $0.529-4.618$ \\
\hline & Consultorio de Enfermeria & 0,468 & 0,410 & $0.395-7.414$ \\
\hline & Otros & 0,752 & 0,044 & $0.595-2.055$ \\
\hline \multirow{2}{*}{$\begin{array}{l}\text { Donde prepara y/o consume el } \\
\text { rehidratante oral }\end{array}$} & Centro de Salud & 0,019 & 0,315 & $1.132-4.345$ \\
\hline & Su a sa & 0,065 & 0,401 & $0.942-5.046$ \\
\hline $\begin{array}{l}\text { Tiempo transcurrido desde la } \\
\text { atención hasta empezar la TRO. }\end{array}$ & $\begin{array}{l}<30 \text { minutos } \\
30 \text { a } 60 \text { minutos } \\
>\text { a } 60 \text { minutos }\end{array}$ & $\begin{array}{l}0,468 \\
0,222 \\
0,377 \\
\end{array}$ & $\begin{array}{l}0,022 \\
0,465 \\
0,330 \\
\end{array}$ & $\begin{array}{l}0.395-7.414 \\
0.685-4.946 \\
0.553-2.017 \\
\end{array}$ \\
\hline
\end{tabular}

Fuente: Proyecto FRAS.R.O. 
la solución preparada de S.R.O. prescrita, no acepta la solución preparada de S.R.O. y preferencia por otros hidratantes orales preparados; el factor institucional no obtuvo relación estadísticamente significativa $r=0,223$ - valor $\mathrm{p} 0,509$.

El resto de variables incluidas en el estudio, en cada una de las dimensiones de factores (socioculturales, personales e institucionales) no tuvieron una correlación estadísticamente significativa con la aceptabilidad de soluc ones rehidratantes orales.

Tabla 3. Factores socioculturales, personales e institucionales relacionados a la aceptabilidad de soluciones rehidratantes orales, en usuarios de establecimientos de salud del Distrito de Comas. LimaPerú 2016

\begin{tabular}{cccc}
\hline FACTORES & $\begin{array}{c}\text { Valor } \\
\mathbf{p}\end{array}$ & $\begin{array}{c}\mathbf{r} \\
\text { spearman }\end{array}$ & IC (95\%) \\
\hline Socioculturales & 0.000 & 0,781 & $4.009-10.238$ \\
Personales & 0,026 & 0,659 & $0.603-1.281$ \\
Institucionales & 0,509 & 0,223 & $0.457-0.951$ \\
\hline
\end{tabular}

Fuente: Proyecto FRAS.R.O.

La tabla 3, evidencia en términos globales, que los factores socioculturales $(r=0,781-p=0,000)$ y los factores personales $(r=0,659-p=0,026)$, presentan correlación estadísticamente significativa a la aceptabilidad de soluciones rehidratantes orales, en usuarios de establecimientos de salud del Distrito de Comas. LimaPerú. Los factores institucionales $(r=0,223-p=0,509)$ no presenta una correlación estadísticamente significativa.

\section{DISCUSIÓN}

La diarrea es una de las enfermedades más prevalentes en el mundo y en el Perú, que afecta predominantemente a los niños, por tanto, son las que requieren con mayor frecuencia atención pediátrica en los primeros niveles de atención de salud como son los Puestos y Centro de Salud. A pesar de eso, ocasionalmente cursa con deshidratación ${ }^{5}$. Al no tener tratamiento etiológico, el tratamiento de elección es la reposición de líquidos y electrolitos para prevenir o tratar la deshidratación ${ }^{6}$. Desde hace años, la rehidratación mas eficiente es mediante las soluciones de rehidratación oral (SRO) ${ }^{6-8}$. En práctica clínica habitual la utilización de estas SRO al comienzo de las diarreas se produce con un doble objetivo: mantener el reposo intestinal, con dieta solida absoluta durante unas horas, y como profilaxis de la deshidratación. Sin embargo, muchos niños rechazan los sueros por su sabor ${ }^{8}$, lo que en algunas ocasiones lleva a la necesidad de rehidratar por vía i.v. El rechazo del SRO hace que por parte de los cuidadores se utilice para el tratamiento o prevención de la deshidratación zumos o soluciones que no cumplen las recomendaciones y que presentan potencial iatrogenia ${ }^{9}$. Existen preparados con presentación en sobres que hay que disolver en diferentes cantidades de agua según el preparado, lo que puede llevar a errores. Por eso se consideran más ventajosas las soluciones de presentación lıquida ${ }^{7}$, porque no necesitan manipulación. Actualmente, existen en el mercado abundantes SRO, a las que se les añaden edulcorantes y saborizantes para incrementar su aceptación. Anteriormente, para mejorar el sabor, se añadían soluciones, como zumos, que incrementaban la osmolaridad y disminuían parte del beneficio de la $\mathrm{SRO}^{10,11}$. En los últimos años se han modificado y sustituido por la adición de diversos saborizantes. A pesar de esto sigue habiendo rechazo a los sueros, y nunca se ha evaluado el beneficio de añadir saborizantes sobre la aceptación de los niños. La palatabilidad o la aceptabilidad en términos más sencillos y adecuados a nuestra realidad a diversos medicamentos, como los antibióticos, se ha analizado en diversas ocasiones, ya que la elección correcta del sabor puede llevar a mejor efectividad ${ }^{12,13}$ al conseguir mejor aceptación y, por tanto, cumplimentación.

En este contexto podemos afirmar a la luz de los hallazgos del presente estudio, donde se evidencia que los factores socioculturales, personales e institucionales relacionados a la aceptabilidad de soluciones rehidratantes orales, en usuarios de establec mientos de salud del Distrito de Comas; son el factor sociocultural $r=0,781$ y valor $p 0,000$, con las variables grado de instrucción secundaria completa y superior, procedencia sierra, ingiere como primera opción soluciones hidratantes preparadas diferentes a las S.R.O. con electrolitos listas para tomar; el factor personal $r=0,659$ - valor $p 0,026$, con las variables edad de 0 a 5 años, no ingiere toda la solución preparada de S.R.O. prescrita, no acepta la solución preparada de S.R.O. y preferencia por otros hidratantes orales preparados; el factor institucional no obtuvo relación estadísticamente significativa $r=0,223$ - valor $p 0,509$; hallazgos que o na erdan o $\mathrm{n}$ las o nb usiones de otros estudios ${ }^{9-13}$, debemos de remarcar que resaltan en el problema de la aceptabilidad a las soluciones rehidratantes orales en personas con enfermedades diarreicas agudas con deshidratación, variables fundamentalmente cualitativas y vinculadas a la percepción de los usuarios respecto al producto y que se debe de tener en cuenta al momento de decidir sobre políticas públicas en salud, relacionado al tipo de productos a adquirir para el tratamiento y control de las enfermedades diarreicas.

El presente estudio presento limitaciones, el tamaño de la muestra y el tipo de muestreo utilizado, ya que el distrito incluido en el estudio tiene una población grande; asimismo es menester manifestar que es necesario otro tipo de estudios de diseño cuasiexperimental y/o experimental que nos permita valorar la aceptabilidad a las soluciones rehidratantes orales por parte de los usuarios a partir de observaciones que se puedan medir más objetivamente en lo que respecta a la aceptabilidad o tolerancia en el mismo acto de la T.R.O. 
Se señalan las siguientes conclusiones:

- Los factores relacionados estadísticamente significativos con la aceptabilidad de soluciones rehidratantes orales, fueron el factor sociocultural: grado de instrucción secundaria completa y superior, procedencia sierra, ingiere como primera opción soluciones hidratantes preparadas diferentes a las S.R.O. con electrolitos listas para tomar y el factor personal: edad de 0 a 5 años, no ingiere toda la S.R.O. para preparar prescrita, acepta la solución de S.R.O. preparada y preferencia por otros hidratantes orales preparados .

- El aporte más importante del presente estudio probablemente es el hallazgo de una de las razones de falla en el tratamiento y prevención de la deshidratación en usuarios con una enfermedad diarreica aguda, sea la no aceptación o rechazo de las S.R.O. a causa del sabor y la aceptabilidad de soluciones hidratantes preparadas diferentes a las S.R.O. con electrolitos listas para tomar, como una opción importante para el tratamiento y prevención de la deshidratación en una EDA, sin embargo es necesario llevar a cabo más estudios que nos permitan establecer con un mayor nivel de confiabilidad y validez la intervención terapéutica más eficaz y eficiente para la prevención y control de las EDAS en el Perú y a partir de la evidencia científica generada implementar políticas públicas en salud costo/efectivas.

Agradecimientos. A todos los usuarios participantes en el estudio atendidos en alguno de los establec mientos de salud de la Red de Salud Túpac Amaru. Diresa Lima Norte.

Conflicto de interés: Los autores declaran no tener algún conflicto de intereses.

Financiamiento: Autofinanciado.

\section{REFERENCIAS BIBLIOGRÁFICAS}

1. Mcllnery TK, Adam HM, Campbell DE, Kamat DM, Kelleher KJ, Hoekelman RA. Tratado de Pediatría. Academia Americana de Pediatría. Ed. Buenos Aires. Editorial médica Panamericana 2011. p 1547-8
2. Enfermedades diarreicas. Organización Mundial de la Salud. 2013. Disponible en: http://www.who.int/ mediacentre/fs330/es/

3. UNICEF. Estado Mundial de la Infancia 1995:29pp.

4. Comunicación personal con Norbert Hirschhorn. Vea también: Riyad S, El Mougi M, Wahsh AA y Hirschhorn N, «After Rehydration: What Happens to the Child?», Journal of Tropical Pediatrics 1991; 37:1-3.

5. San Emeterio G J, Rubiol C, Álvarez M. Tratamiento de la diarrea aguda infantil en atención primaria. Inf Ter Sist Nac Salud. 1998;22:109-16.

6. Riechmann R, Torres B. Diarrea aguda. En: AEP, editor. Protocolos diagnósticos y terapéuticos. [citado 4 Jun 2009]. Disponible en: URL: http://www.aeped.es/protocolos/ gastroentero/2.pdf.

7. Hartling L, Bellemare S, Wiebe N, Russell K, Klassen TP, Craig W. Oral versus intravenous rehydration for treating dehy ration due to gastroenteritis in $\mathrm{b}$ ildren. Cob rane Database Syst Rev. 2006;3:CD004390.

8. Calatayud A, Martínez M, Fernández B, Fernández $P$, editors. Manual de Rehidratación Oral. Sociedad Española de Urgencias de Pediatría; Ediciones JB: 2000.

9. Herranz Jordán HB, Torres-Pardo Muñoz P. Aceptación de sabor de una nueva solución de rehidratación oral en niños sanos. Pediatr Aten Primaria. 2000;2:25-30.

10. Vitoria I, Castells E, Ochoa E, Arias T. Rehidratación oral con bebidas refrescantes. Riesgo de yatrogenia en la infancia. An Esp Pediatr. 2001;54:24-5.

11. Te Loo DM, Van der Graaf F, Ten WT. The effect of flavoring oral rehydration solution on its composition and palatability. $J$ Pediatr Gastroenterol Nutr. 2004;39:545-8.

12. Powers JI, Gooch M, Oddo LP. Comparison of the palatability of the oral suspension of cefdinir vs. amoxicillin/clavulanate potassium, cefprozil and azithromycin in pediatric patients. Pediatr Infect Dis J. 2000;19:S174-80.

13. Angelilli ML, Toscani M, Matsui DM, Rieder MJ. Palatability of oral antibiotics among children in an urban primary care center. Arch Pediatr Adolesc Med. 2000;154:267-70.

\section{Correspondencia:}

Walter Gómez Gonzales. Universidad Alas Peruanas, Escuela de Post Grado. Av. Cayetano Heredia 1044, Jesus Maria 15072. Lima-Perú, (+51) 944578766.

E-mail:w_gomez go@doc.uap.edu.pe waltergomez29@yahoo.com 